\title{
Nonlinear structural behavior and vibration control of a double curved cable net under the dynamic excitations
}

\author{
Mohammad Khalkhalihaa (D), Panam Zarfama* (D), Raham Zarfam ${ }^{\text {b }}$ \\ a Department of Civil Engineering, Science and Research Branch, Islamic Azad University, Tehran, Iran. Email: khalkhaliha_m@yahoo.com, \\ zarfam@srbiau.ac.ir \\ b Leonhardt, Andrä und Partner, Stuttgart, Germany. Email: raham.zarfam@lap-consult.com \\ * Corresponding author
}

http://dx.doi.org/10.1590/1679-78255884

\begin{abstract}
The paper is regarding to the structural dynamics of a saddle shaped cable-net structure with two high points and two low points at the corners. The net cables are pre-stressed by stretching the four edge cables that are supported at the corner points. A series of parametric nonlinear dynamic analysis is utilized to evaluate the dynamic response of the system under the different structural masses and geometries, pretensions and alignments of cables and the amplitudes of dynamic excitation. An equivalent single-degree-of-freedom model is presented as a simplified method to estimate the dynamic response of the full finite element model. The simulation results show that the equivalent model can accurately estimate the displacement response of multi-degree-of-freedom model under dynamic excitation. Furthermore, a nonlinear active control algorithm is applied to decrease the structural response under the transient wind excitation and the results indicate the effectiveness of the proposed control algorithm.
\end{abstract}

\section{Keywords}

Cable-net structure, active control, nonlinear dynamic analysis, geometrically nonlinear, equivalent SDOF, response spectrum

\section{Graphical Abstract}

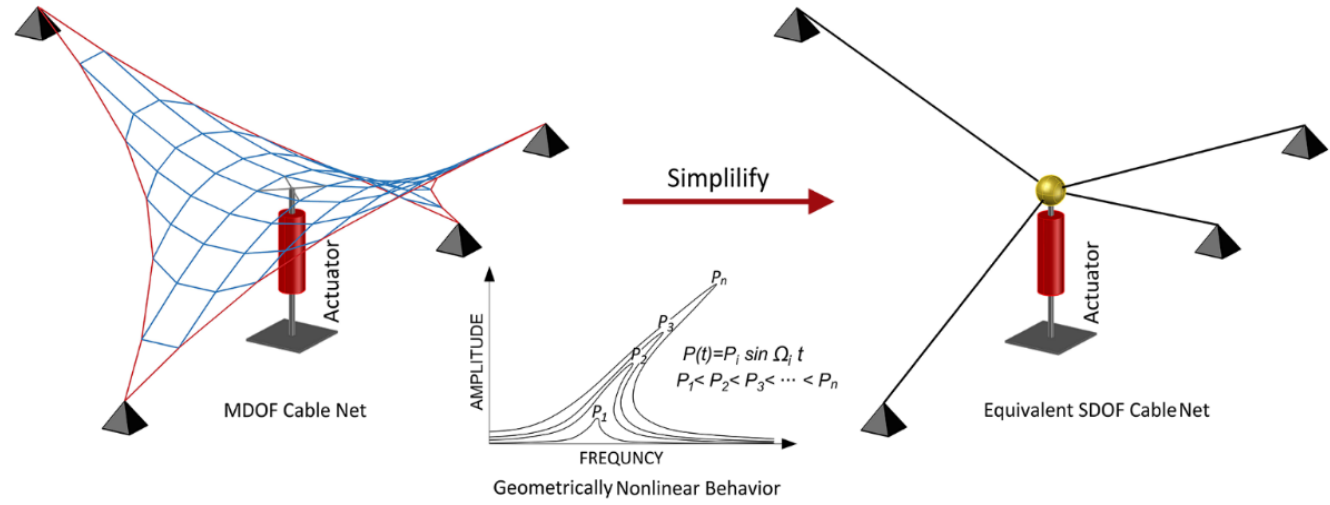




\section{INTRODUCTION}

During the last decades, cable nets have been widely applied in structural systems due to their desired features of high tensile strength, lightweight, and appealing architectural shapes, which can be utilized to cover large spans and in glass facades. The cable nets often experience large deformations during the transfer of loads to the supports. The inherent characteristics of such structures are geometric nonlinearity, which makes the stiffness of the system interact its distorted state, which is mainly in contrast with the conventional ones (Levy and Spillers, 2003). Several techniques have been introduced for tackling geometric nonlinearity problems based on Newton's methods, including the ones described in (Papadrakakis and Gantes, 1988; Papadrakakis and Balopoulos, 1991; Waziri et al., 2010). Since cable nets are a kind of flexible structures, which possess characteristics such as low stiffness and weight, weak damping ratio and low and closely spaced resonant frequencies (Haiyan et al., 2013), they are susceptible to dynamic loadings, such as wind, that may make them to have considerable levels of vibration. Therefore, one of the most important issues in designing of such structures is the recognition of their dynamic behavior. On the other hand, the effects of geometric nonlinearity and large deformations in cable nets increase the complexity of their dynamic response calculation.

Pre-stressed cable-net roofs are capable to produce various shapes. The most common shape is the hyperbolic paraboloid. Stefanou (1992) presented an approach for dynamic analysis of cable nets subjected to wind excitation. The proposed approach, which is established by minimizing the total potential energy, is based on a systematic response calculation in the time domain of the dynamic forces at the end of each time step. Kwan (2000) derived a geometrical nonlinear stiffness matrix for a three-dimensional pre-stressed cable element based on the flexibility method. He also presented a simple technique for determining the natural frequencies of the cable structures, neglecting the nonlinear stiffness terms. Gambhir and Batchelor $(1977,1979)$ presented a finite element model to study the dynamic behavior of the three-dimensional cable nets in which, the stiffness and consistent mass matrices of the pre-stressed cable elements were derived using the principle of virtual work in its deformed form. Talvik (2001) suggested a mathematical model for static and dynamic analysis of pre-stressed cable nets with the flexible contour ring. Lazzari et al. (2001) investigated the nonlinear dynamical behavior of an actual saddle-form cable net subjected to wind excitation, by means of a finite element approach. They showed that the increase in deformation causes changes in the stiffness and the resonance frequency. Therefore, this phenomenon prevents the continuous increase of the vibration amplitude, which occurs for linear systems. Zingoni (1996) introduced a computational scheme for vibration analysis of flat cable nets comprising of highly tensioned cables and having orthogonal projections in the plan. It was assumed that the cable nets are under the inertial forces centered on the intersection of the cables. Vassilopoulou and Gantes $(2005,2010,2011,2012,2013,2016)$ conducted a research on the dynamic behavior of saddle form cable nets, having a circular plan view. They explored the nonlinear dynamic phenomena in a single-degree-of-freedom (SDOF) model of cable net, and also proposed semi-empirical formulae to estimate the frequencies of the first vibration modes of the multi-degree-of-freedom (MDOF) cable net with rigid and flexible edge rings.

The form finding, which generally used to find the optimal form of membrane structures, is conducted in a finite element method and takes into account the pre-stress of each element and its slight stiffness. Such behavior is in accordance with soap films (Haug and Powell, 1972). Bletzinger and Ramm (2001) examined three different computational methods of form finding of lightweight structures, including (a) simulation of hanging models, (b) numerical simulation of soap films, and (c) structural shape optimization. Haug and Powell (1972) performed a finite element procedure for the form finding of cable nets and presented results for three different types of cable nets. The initial geometry of the cable net was obtained from sketches. By changing the pre-stress and/or changing the unstressed length of the members, it would be possible to adjust the shape of a favorite configuration. Nonlinear structure analysis using Newton-Raphson iteration was used for obtaining a new equilibrium shape following each set of adjustments. Birnstiel (1972) described some mathematical techniques for determining the initial shape of cable structures and calculating their displacement due to static loads, taking into account the geometric nonlinearity. Atai and Mioduchowski (1998) derived equilibrium equations of cable nets with elasto-plastic behavior, considering the history of loading and slackening of cables.

Monolayer cable net system supporting glass facades is a flat cable net that is physically sensitive to wind excitations. Shi et al. (2007) showed that the geometric shape and pretension have a significant effect on the stiffness of such structures. However, with increasing the vertical displacement of the central node of the cable net, the influence of geometric shape is far greater than that of pretension. Wu et al. (2010) examined the wind-induced response of Monolayer cable nets in frequency-domain method. Feng et al. (2013) determined the nonlinear vibration differential equation and vibration frequency of cable net glazing subjected to earthquake loading and established a geometrically nonlinear SDOF model for cable net glazing. 
One of the most important issues in designing of cable nets is optimization, which addresses two issues of safe and economic design at the same time. The optimization of the cable nets can be done through control systems. Bleicher et al. (2011) proposed an active vibration control system for a light and flexible stress ribbon footbridge using pneumatic muscle actuators. Wu et al. (2016) studied the nonlinear active vibration control of cable net structures, using piezoelectric stacked actuators. They demonstrated that nonlinear control method enhanced the performance of the system by decreasing the vibration to a lower amplitude within a shorter time.

In this paper, with a parametric investigation of the dynamic behavior of saddle shaped cable-net structures, a simplified method is proposed which reduces the expensive cost of geometrical nonlinear dynamic analysis of such complex structures. By this means, the influence of each parameter such as the mass and geometric of structure, pre-stress and alignment of cables, amplitude of oscillation on the main factors of the dynamic behavior of the structure is clarified. Then, by introducing an equivalent SDOF system representing the main structure, the effectivity of the particle swarm optimization (PSO) algorithm as an active control method is proven that provides one further step to apply nonlinear active control systems on such structures.

\section{The definition of the structural model}

In order to simplify and cover a wide range of dynamic behaviors and parameters affecting them such as: geometric, structural mass, pre-stress and alignment of cables and amplitude of oscillation; a simplified saddle shaped cable-net model with two high points and two low points at the corners and a square plan view with dimensions of $5 \mathrm{~m} \times 5 \mathrm{~m}$, which consists of two types of the edge cables and the cable net is used for the parametric study. The net cables are pre-stressed by stretching four edge cables that are supported at corner points (Figure 1). The PE7 cable with the cross-sectional area of $60 \mathrm{~mm}^{2}$ and the PE15 cable with the cross-sectional area of $117 \mathrm{~mm}^{2}$ according to the Germany DIN EN 12385-Stainless Steel (ETA, 2015) are considered for the edge cables and net cables, respectively. The elastic modulus of cables is $130 \mathrm{GPa}$.
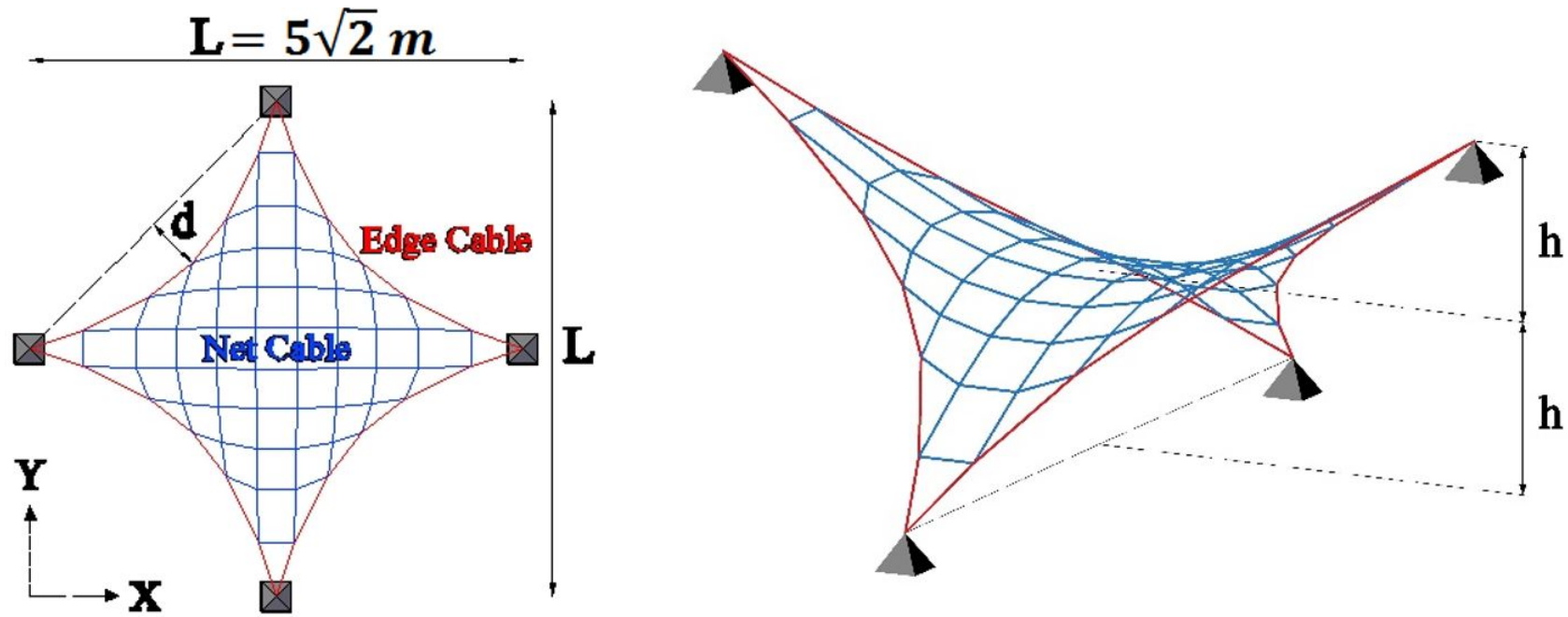

Figure 1 Saddle shaped cable-net structure with two high points and two low points at the corners.

The geometric shape of the selected model is determined by using form-finding analysis, which is influenced by factors such as the coordinates of fixed points and the ratio of the edge cable pretension to the net cable pretension $\left(R=T_{0, e} / T_{0, n}\right)$. The rise $(h)$ of the cable net is explained as the difference of height between the highest or lowest supports and the center point.

A finite element model is developed for nonlinear dynamic analysis in MATLAB software. The three-dimensional corotational truss is used to model the cable element, which is allowed to have arbitrarily large displacements and rotations at the global level. All elements are assumed to remain linear elastic and without compression resistance. Newmark's direct integration method and Newton-Raphson's method are employed to solve the nonlinear equation of motion.

As shown in Figure 2, the displacement and tension responses obtained from the finite element model are compared with those generated by OpenSees (McKenna et al., 2000) to validate the numerical modelling. The compared model is a saddle-shaped cable-net with $\zeta$ (damping ratio) $=2 \%, M$ (total structural mass) $=233 \mathrm{~kg}$ and $T_{0, n}$ (net cable pretension) $=1 \mathrm{kN}, h=0.5 \mathrm{~m}$ and $R=10$ subjected to wind excitation (Zarfam et al., 2017) shown in Figure 3 . Comparing the responses proves that the numerical modelling can provide accurate results. 


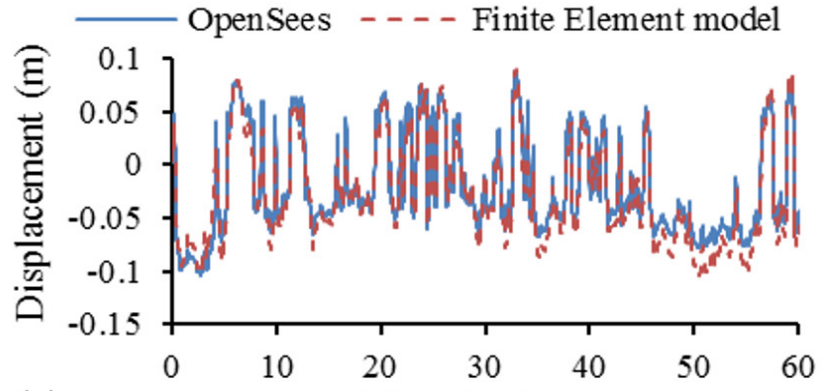

(a) Time (sec)

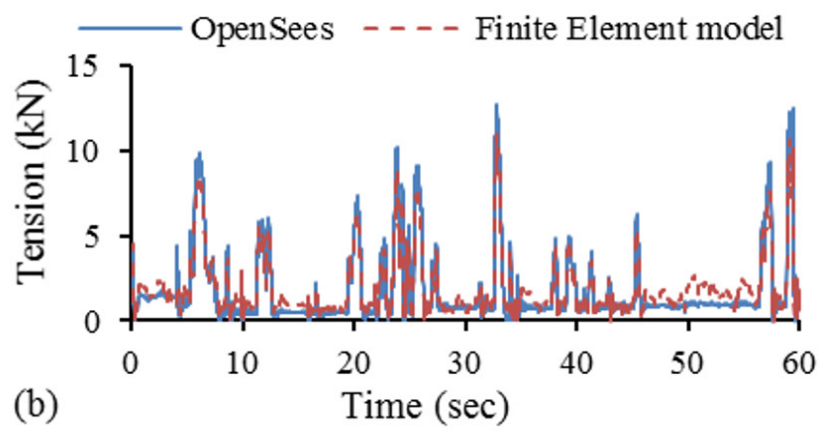

(b)

Figure 2 Comparison of the responses of the cable net subjected to wind excitation calculated by the OpenSees software and finite element method; (a) displacement response at the center point and (b) tension response of net cable.

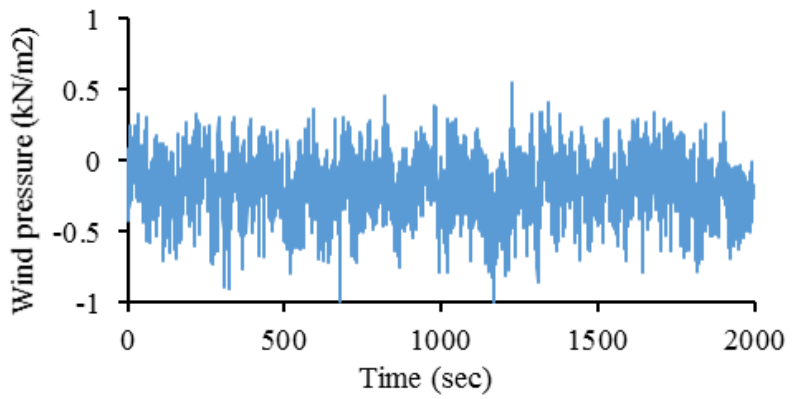

Figure 3 Time-history of the wind pressure (Zarfam et al., 2017).

\section{Parametric investigation on the dynamic behavior of the cable net}

In order to investigate the geometrical effects on the dynamic behavior of the cable net, five different rises and three different pretension ratios are considered as $h=\{0,0.25,0.5,1$ and $1.5 \mathrm{~m}\}$ and $R=\{10,20$ and 30$\}$, respectively. It covers a variety range of the rise/span ratios as $h / L=\{$ from 0 to $1.5 / 5 \sqrt{2}\}$ that can be used in practical situations. In addition, six pretension levels for net cables are considered as $T_{0, n}=\{0.1,1,2,3,4$ and $6 \mathrm{kN}\}$.

The cable-net models without and with acrylic sheet, wooden and lightweight concrete covering panel are considered for investigating the effect of mass on dynamic behavior. Table 1 represents the specifications of the covering panels, according to Eurocode 1 (2002), and the total structural mass $(M)$.

Table 1 The specifications of the covering panels and the total structural mass $(M)$.

\begin{tabular}{cccc}
\hline \multirow{2}{*}{ Panel material } & Thickness & Specific weight & Total structural mass $(\boldsymbol{M})$ \\
\cline { 2 - 4 } & $m$ & $\mathrm{kN} / \mathrm{m}^{3}$ & $\mathrm{~kg}$ \\
\hline None & - & - & 61 \\
Acrylic sheet & 0.004 & 12.0 & 233 \\
Wood & 0.03 & 8.0 & 852 \\
Lightweight concrete & 0.05 & 12.0 & 2000 \\
\hline
\end{tabular}

\subsection{The study of the geometric, pretension and mass effects}

The undamped free vibration is conducted with initial vertical displacement $u(0)=0.3 \mathrm{~m}$ in center point of the cable net. The comparison between the dominant frequency of undamped cable net models with different parameters is shown in Figure 4. Since cable structures have geometrically nonlinear behavior, the dominant frequency is determined by transforming the displacement response of the central node of the cable net from time domain to frequency domain, using Fast Fourier Transform (FFT). 

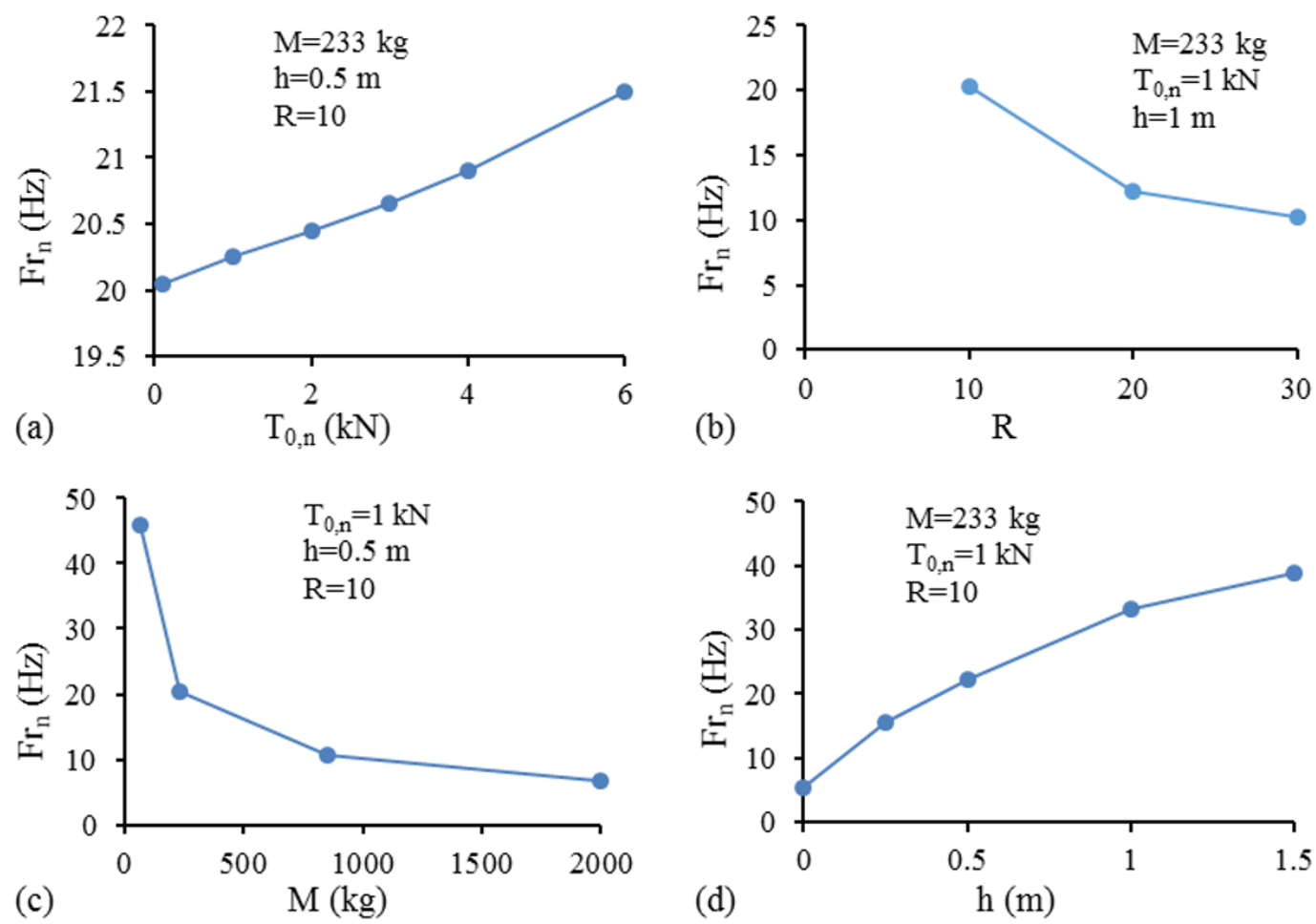

Figure 4 The dominant frequency of undamped cable net models vs. (a) the pretension of the net cable, (b) the ratio of the edge cable pretension to the net cable pretension, (c) the total structural mass and (d) the rise of the cable net.

Figure 4(a) illustrates that the frequency of the system increases linearly by increasing pretension of the cables, which indicates an increase in stiffness of the cable net. On the other hand, by increasing the cable pretension, highergrade cable should be used which leads to higher costs.

As shown in Figure $4(\mathrm{~b})$, an increase in the pre-tension ratio $(R)$ reduces the frequency, indicating a decrease in system stiffness. Because, the more the pre-tension ratio increases, the more the pre-tension of the edge cables that act as the spring support increases. By increasing the pretension ratio, the pretension of edge cables increases, so their horizontal sag $(d)$ reduces and the length of the net cables enlarges. These two geometrical effects in the form-finding phase cause more flexibility in the system.

The chart of dominant frequency vs structural mass has shown in Figure 4(c). It indicates that the system frequency is inversely proportional to the square root of the structural mass.

Figure 4 (d) depicts that by increasing the rise, the dominant frequency of the system increases. Since by increasing the rise, the vertical component of the internal resistance forces of the cables increases, consequently the vertical stiffness of the cable net increases. On the other hand, when the alignment of the net cables is chosen according to Figure 5(a), as shown in Figure 5(b), the rise does not significantly affect the frequency of the system. In curved cable nets, the curvature of the net is diagonally in the direction of the same level supports. Since the direction of the cables are not aligned to the curvature of the net, the cables lack curvature and the cable net acts like a flat net.
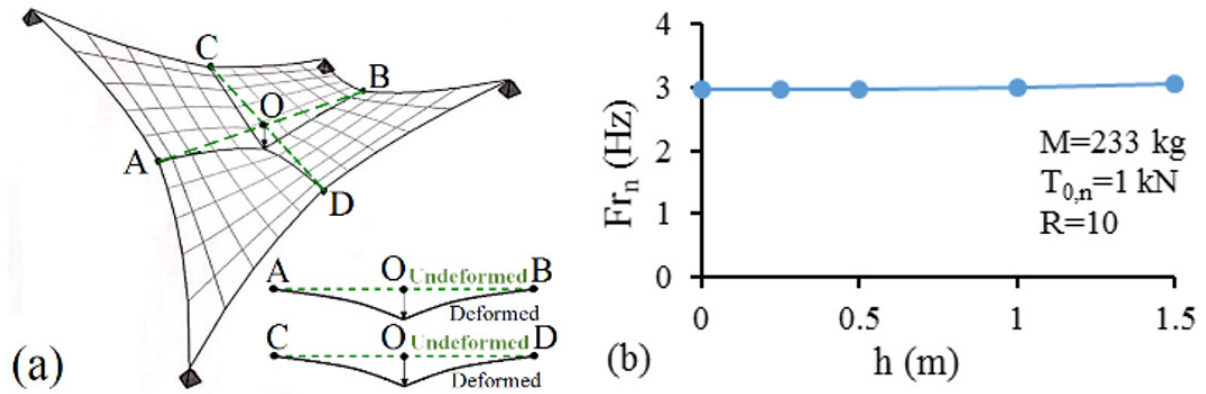

Figure 5 Cable net with alignment of incorrect cables, (a) configuration and (b) dominant frequency vs. rise. 


\subsection{The study of the oscillation amplitude effects}

The comparison between the time-history diagrams of the central node displacement of cable net models with $h=0$ and $1.5 \mathrm{~m}$ under the undamped free vibration with initial vertical displacement of $u(0)=0.1$ and $0.3 \mathrm{~m}$ are shown in Figure 6. By increasing the oscillation amplitude, the fundamental frequency of system with $h=0$ and $1.5 \mathrm{~m}$ increases from 2.6 and $14.1 \mathrm{~Hz}$ to 5.25 and $38.75 \mathrm{~Hz}$, respectively. On the other hand, it is observed that by increasing the rise, the oscillation amplitude decreases significantly due to the mode cancellation; which means, by increasing the rise in this type of saddle shaped cable-net structure, the higher-frequency modes are activated and the effect of lower-frequency modes are cancelled. Therefore, the structure can't oscillate on the main mode (Figure 7).

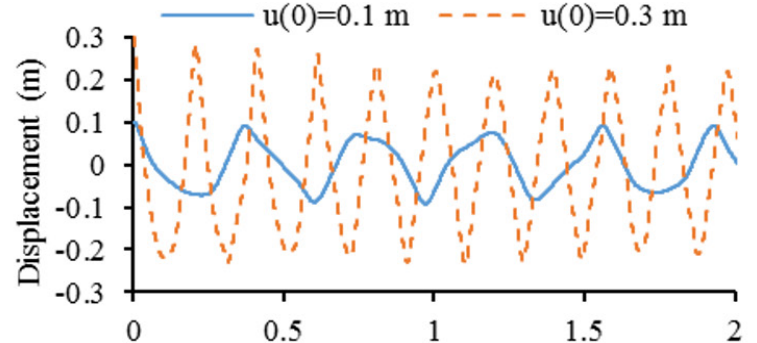

(a)

Time (sec)

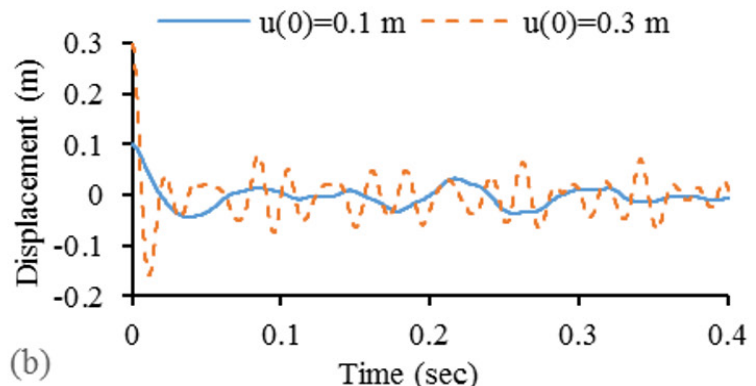

Figure 6 Free vibration of undamped cable net with (a) $h=0$ and (b) $h=1.5 \mathrm{~m}$.
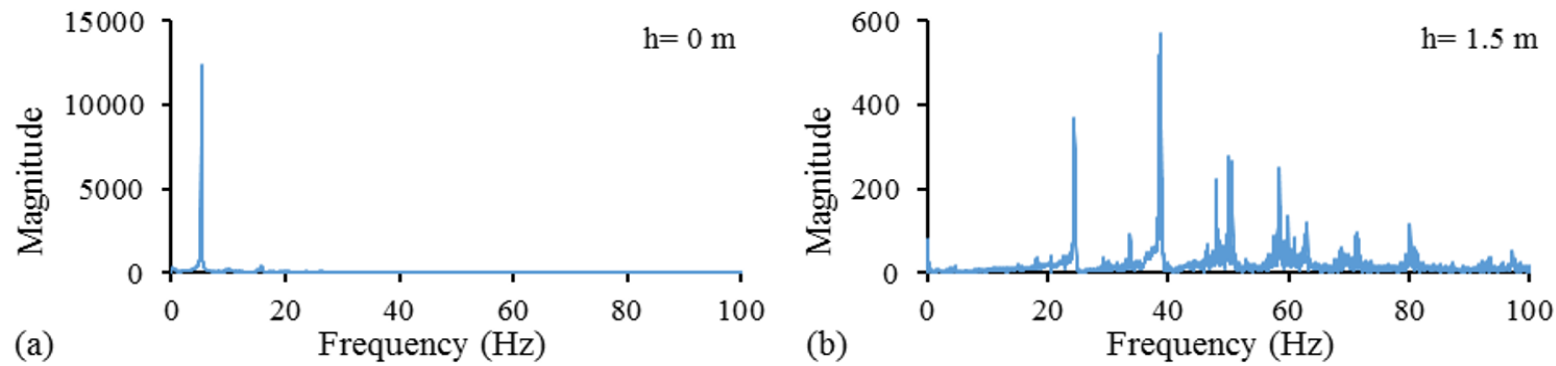

Figure 7 Fourier spectrum of the undamped cable net with (a) $h=0$ and (b) $h=1.5 \mathrm{~m}$.

\subsection{Response spectrum}

The displacement response spectrum of undamped cable net with $h=0.5 \mathrm{~m}, R=10, T_{0, n}=1 \mathrm{kN}$ and $M=233 \mathrm{~kg}$ to vertical harmonic excitation $P(t)=P_{0} \sin (\Omega t)$ is presented in Figure 8. Five exciting amplitudes are considered as $P_{0}$ equal to $10 w, 20 w, 30 w, 40 w$ and $50 w$, in which, $w$ is the total weight of the structure and $\Omega$ is the exciting frequency from 10 to $30 \mathrm{~Hz}$ with $0.1 \mathrm{~Hz}$ interval.

The more the amplitude of dynamic excitation rises, the more the dominant frequency of the system corresponding to the peak of the response spectrum increases and tends to right. Therefore, the frequency of the system depends not only on the stiffness and mass, but also to the amplitude of the dynamic excitation. As the amplitude increases, the average axial force of members increases, leading to about $30 \%$ increase at the vibrational frequency. This represents the geometrically nonlinear behavior of cable net systems, in which, the response is not only depended on the excitation frequency, but also to the amplitude of the excitation.

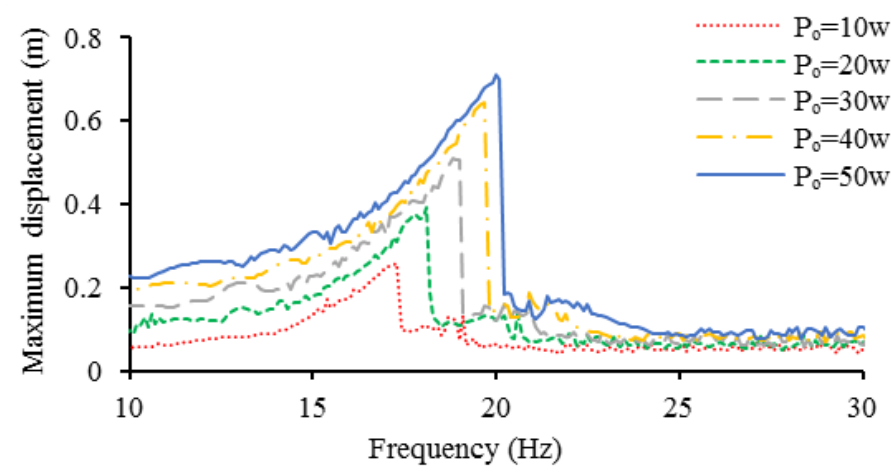

Figure 8 Displacement response spectrum of undamped cable net to harmonic vibration with different exciting amplitudes. 


\subsection{Response to wind excitation}

The models were subjected to wind excitation with the time history presented in Figure 3 . The damping ratio of $2 \%$ was applied to assess more realistic behavior of the system subjected to the wind excitation (Vassilopoulou and Gantes, 2011; Feng et al., 2013).

Comparing the displacement response factor $R_{d}$ of damped cable net models with different rises under the wind excitation, shown in Figure 9, if $h>0$ then $R_{d}<1$, that is, the dynamic displacement amplitude is less than the static displacement. The $R_{d}$ is the ratio of the maximum of the dynamic displacement $u_{\text {max }}$ to the static displacement $u_{s t}$. Because, the more the rise increases, the more the frequency of the system increases and gets away from the dominant frequency-range of wind excitation with lower frequency-range. It shows the importance of dynamic analysis of flat cable nets, comparing to the ones with rise.

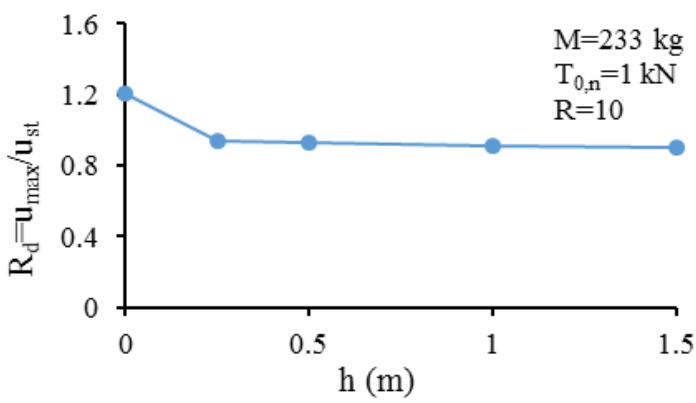

Figure 9 Displacement response factor of cable net vs. rise.

\section{Equivalent SDOF modeling}

The dynamics of systems with geometric nonlinearity, which their stiffness depends on the deformation, subjected to large amplitudes can be well described by a cubic hardening nonlinearity (Setio et al., 1992a, 1992b). Kung and Pao (1972), Parzygnat and Pao (1978) and Nayfeh and Mook (1979) showed that such nonlinear equations can be reduced to the equation of motion of Duffing's oscillator. Perturbation techniques such as: Lindstedt's, multiple scales, averaging, harmonic balance methods and etc. are used as the solution of nonlinear equations of the motions (Nayfeh and Mook, 1979; Vakakis, 2002; Kevorkian and Cole, 1996; Jager and Furu, 1996; Rand, 2005). The solution of nonlinear differential equations can be obtained relatively simple by numerical integration method such as Runge-Kutta method (Kreysizig, 2011). However, this procedure is unreasonably prolonged to obtain a stationary solution of a MDOF system. Therefore, an equivalent SDOF model is introduced to evaluate the dynamic amplifications of the MDOF cable net. The SDOF model consists of two crossing members of pre-stressed fix-ended cables with opposite concavities (Figure 10). Only the degree of freedom is considered along the z-axis of the central node. The cable material is treated as linear elastic in tension, without compression resistant. Both cables have the same size of span $(L)$, rise $(h)$, cross-section area $(A)$, elastic modulus $(E)$ and initial strain $\left(\varepsilon_{0}\right)$. According to Hooke's law $\varepsilon_{0}=T_{0} / E A$ where $T_{0}$ is the cable pretension. The total uniformly distributed mass of the structure is considered as the lumped mass $(M)$ attached to the central node. Considering vertical displacement of the central node as $u(t)$, the equation of motion along the $z$-axis and subjected to a dynamic load $P_{z}(t)$ can be written as:

$M \ddot{u}(t)+C \dot{u}(t)+T_{z}(t)=P_{z}(t)$

$T_{z}(t)$ is the sum of the tension for each deformed cable element $\left(T_{i}(t), i=1,2,3\right.$ and 4$)$ at the central node, along the $z$ global axis with considering the large deformations as (Vassilopoulou and Gantes, 2011):

$T_{Z}(t)=\sum_{i=1}^{4} T_{i z}(t)=2\{\frac{\overbrace{E A\left(\frac{l_{1,2}(t)-l_{0}}{l_{0}}\right)}^{T_{1,2}(t)}}{\left(\frac{h+u(t)}{l_{1,2}(t)}\right)}-\overbrace{E A\left(\frac{l_{3,4}(t)-l_{0}}{l_{0}}\right)}^{T_{3,4}(t)}\left(\frac{h-u(t)}{l_{3,4}(t)}\right)\}$

$l_{0}$, the initial length of each cable element, is expressed as: 
$\varepsilon_{0}=\frac{l_{T}-l_{0}}{l_{0}} \Rightarrow l_{0}=\frac{l_{T}}{1+\varepsilon_{0}}$

$l_{T}$, the initial pretensioned length of each cable element, is defined as:

$l_{T}=\sqrt{(L / 2)^{2}+h^{2}}$

$l_{i}(t), i=1,2,3$ and 4 , the deformed length of the $i^{\text {th }}$ cable element, is written as:

$l_{1,2}(t)=\sqrt{(L / 2)^{2}+(h+u(t))^{2}}, l_{3,4}(t)=\sqrt{(L / 2)^{2}+(h-u(t))^{2}}$

Substituting the Eqs. (3) - (5) into the Eq. (2), $T_{z}(t)$ becomes:

$T_{Z}(t)=E A\left\{\frac{4 u\left(1+\varepsilon_{0}\right)}{\sqrt{(L / 2)^{2}+h^{2}}}+\frac{2(h-u(t))}{\sqrt{(L / 2)^{2}+(h-u(t))^{2}}}-\frac{2(h+u(t))}{\sqrt{(L / 2)^{2}+(h+u(t))^{2}}}\right\}$

and $C$ is the damping coefficient, expressed as:

$C=2 \zeta M \omega_{z 0}$

where $\zeta$ is the damping ratio and $\omega_{z 0}$ is the natural frequency for the undeformed state, defined as (Vassilopoulou and Gantes, 2011):

$\omega_{z}(t)=\sqrt{\frac{1}{M} \frac{\partial T_{z}(t)}{\partial u(t)}} \stackrel{u(t)=0}{=} \omega_{z 0}=\sqrt{\frac{4 E A}{M} \cdot \frac{\left(h^{2}+\varepsilon_{0}(L / 2)^{2}+\varepsilon_{0} h^{2}\right)}{\sqrt{\left((L / 2)^{2}+h^{2}\right)^{3}}}}$

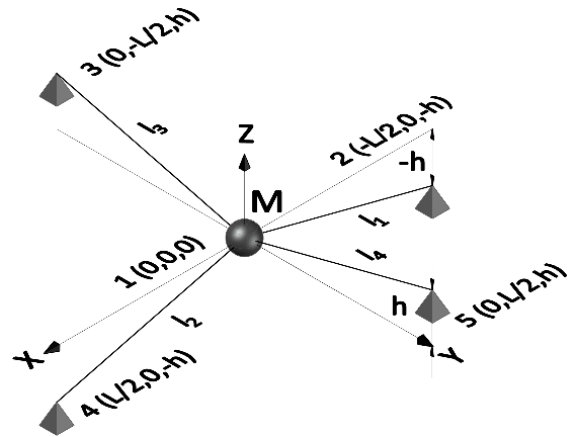

Figure 10 Geometric shape of SDOF cable net model.

Assuming $\phi(x . y)=A \sin (\pi x / L) \sin (\pi y / L)$ as the first eigen mode shape of the net, the equivalent mass $\left(M^{*}\right)$ and the equivalent force $\left(P^{*}\right)$ are estimated as: Eq. (9) and Eq. (10), respectively.

$M^{*}=\sum_{i=1}^{n} m_{i} \sin ^{2} \frac{\pi x_{i}}{L} \sin ^{2} \frac{\pi y_{i}}{L}$

$P^{*}=\sum_{i=1}^{n} p_{i} \sin \frac{\pi x_{i}}{L} \sin \frac{\pi y_{i}}{L}$

where $m_{i}$ and $p_{i}$ are the lumped mass and the exciting amplitude at the $i^{\text {th }}$ node of the full FE model, respectively. $n$ is number of cables of the full FE model. $x_{i}$ and $y_{i}$ are the coordinates of the $i^{\text {th }}$ node of the full FE model (Figure 1).

Assuming the span, rise and initial strain of SDOF model as same as the full FE model, the equivalent axial stiffness $(E A)^{*}$ is calculated by using the following equation:

$(E A)^{*}=\frac{P^{*}}{\left\{\frac{4 u_{S t}\left(1+\varepsilon_{0}\right)}{\sqrt{(L / 2)^{2}+h^{2}}}+\frac{2\left(h-u_{S t}\right)}{\sqrt{(L / 2)^{2}+\left(h-u_{S t}\right)^{2}}}-\frac{2\left(h+u_{S t}\right)}{\sqrt{(L / 2)^{2}+\left(h+u_{S t}\right)^{2}}}\right\}}$ 
where $u_{s t}$ is the static displacement of central node of the full FE model under the dynamic excitation amplitude. For the equivalent SDOF system, $2 \%$ extra damping ratio is considered to equate its kinetic energy with the kinetic energy of full FE structure.

As shown in Figure 11, a comparison between displacement response spectrum of the equivalent SDOF and full FE models with $h=0$ and $0.5 \mathrm{~m}$ under the vertical harmonic excitation $P(t)=P_{0} \sin (\Omega t)$ with $P_{0}=10 \mathrm{w}$ is considered to verify the accuracy of this procedure. Where $w$ is the total weight of the full FE model and $\Omega$ is the exciting frequency from 0.1 to $50 \mathrm{~Hz}$ with $0.1 \mathrm{~Hz}$ interval. The parameters of the full FE models and the calculated parameters of the equivalent SDOF models are presented in Table 2. The equivalent SDOF model has estimated the resonance frequency of the full FE models with $h=0$ and $0.5 \mathrm{~m}$ with a deviation of $3.1 \%$ and $4.6 \%$, respectively.

Table 2 The parameters of the full FE and equivalent SDOF models under the harmonic excitation.

\begin{tabular}{|c|c|c|c|c|c|c|c|c|}
\hline \multicolumn{9}{|c|}{ Parameters of full FE model } \\
\hline $\boldsymbol{h}$ & $\Sigma m_{i}$ & $\Sigma p_{i}$ & $\boldsymbol{E A}$ & $T_{0, n}$ & $\boldsymbol{R}$ & $\varepsilon_{0, n}=T_{0, n} / E A$ & $\boldsymbol{L}$ & $\zeta$ \\
\hline$m$ & $k g$ & $k N$ & $k N$ & $k N$ & - & - & $m$ & $\%$ \\
\hline 0 & 233 & 22.43 & $7.80 \times 10^{3}$ & 1.0 & 10 & $1.28 \times 10^{-4}$ & $5 \sqrt{2}$ & 0 \\
\hline 0.5 & 233 & 22.43 & $7.80 \times 10^{3}$ & 1.0 & 10 & $1.28 \times 10^{-4}$ & $5 \sqrt{2}$ & 0 \\
\hline \multicolumn{9}{|c|}{ Parameters of equivalent SDOF model } \\
\hline $\boldsymbol{h}$ & $M^{*}$ & $\boldsymbol{P}^{*}$ & $(\boldsymbol{E A})^{*}$ & $T_{0}=$ & & $\varepsilon_{0}$ & $\boldsymbol{L}$ & $\zeta$ \\
\hline$m$ & $k g$ & $k N$ & $k N$ & & & - & $m$ & $\%$ \\
\hline 0 & 179.7 & 19.77 & $4.78 \times 10^{4}$ & & & $1.28 \times 10^{-4}$ & $5 \sqrt{2}$ & 2 \\
\hline 0.5 & 179.7 & 19.77 & $8.27 \times 10^{4}$ & & & $1.28 \times 10^{-4}$ & $5 \sqrt{2}$ & 2 \\
\hline
\end{tabular}
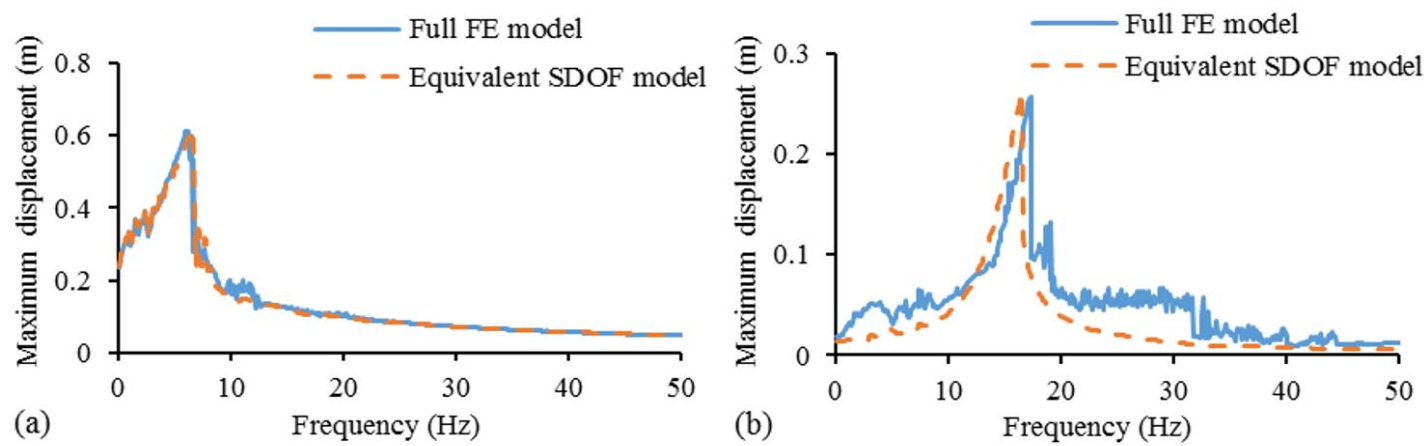

Figure 11 Comparison of the displacement response spectrum of the full FE and equivalent SDOF models under the harmonic excitation; (a) $h=0$, (b) $h=0.5 \mathrm{~m}$.

The efficiency of the calibrated equivalent model has been evaluated under the wind load, presented in Figure 3 . The parameters of the full FE model and the calculated parameters of the equivalent SDOF model are presented in Table 3. The equivalent SDOF model estimates the dynamic response of the full FE model under the wind excitation with $2.76 \%$ deviation (Figure 12).

Table 3 The parameters of the full FE and equivalent SDOF models under the wind excitation.

\begin{tabular}{|c|c|c|c|c|c|c|c|c|}
\hline \multicolumn{9}{|c|}{ Parameters of full FE model } \\
\hline $\boldsymbol{h}$ & $\Sigma m_{i}$ & $\Sigma p_{i}$ & $E \boldsymbol{A}$ & $T_{0, n}$ & $\boldsymbol{R}$ & $\varepsilon_{0, n}=T_{0, n} / E A$ & $L$ & $\zeta$ \\
\hline$m$ & $k g$ & $k N$ & $k N$ & $k N$ & - & - & $m$ & $\%$ \\
\hline 0 & 233 & 8.5 & $7.80 \times 10^{3}$ & 1.0 & 10 & $1.28 \times 10^{-4}$ & $5 \sqrt{2}$ & 0 \\
\hline \multicolumn{9}{|c|}{ Parameters of equivalent SDOF model } \\
\hline $\boldsymbol{h}$ & $M^{*}$ & $\boldsymbol{P}^{*}$ & $(\boldsymbol{E} \boldsymbol{A})^{*}$ & $T_{0}=$ & & $\varepsilon_{0}$ & $L$ & $\zeta$ \\
\hline$m$ & $k g$ & $k N$ & $k N$ & & & - & $m$ & $\%$ \\
\hline 0 & 179.7 & 7.52 & $4.95 \times 10^{4}$ & & & $1.28 \times 10^{-4}$ & $5 \sqrt{2}$ & 2 \\
\hline
\end{tabular}




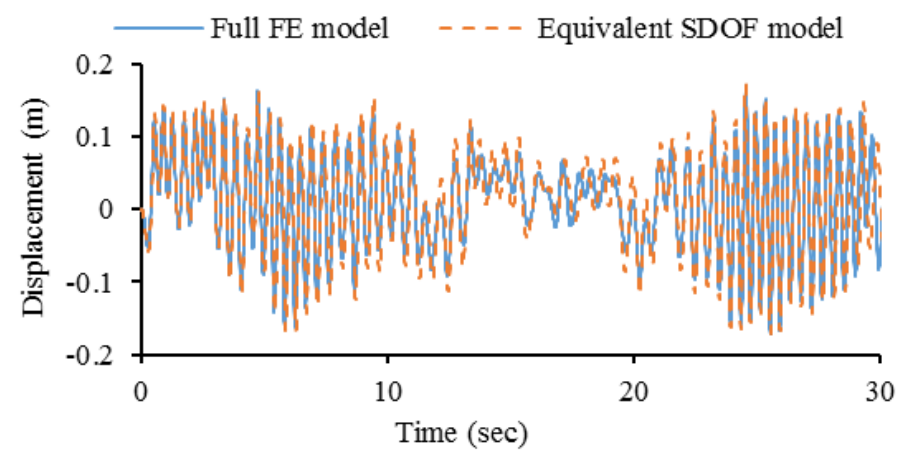

Figure 12 Comparison of the displacement response of the full FE and equivalent SDOF models under the wind excitation.

\section{The application of active control to improve cable net behavior}

In order to reduce the dynamic response of the mentioned cable net with the geometrical nonlinear behavior described in previous sections, PSO is applied as control algorithm. Since the purpose of this part is to verity the efficiency of the control algorithm, using the equivalent SDOF method is suitable to reach this goal (Figure 13). The nonlinear equation of motion of the controlled SDOF system can be written as:

$M \ddot{u}(t)+C \dot{u}(t)+T_{z}(t)+T_{\text {control }}(t)=P_{z}(t)$

where $T_{\text {control }}(t)$, an applied control force to the central node and along the $z$ global axis, is expressed as (Soong, 1990):

$T_{\text {control }}(t)=G(t) X(t)$

$X(t)$, the state vector, is expressed as:

$X(t)=\{u(t) \quad \dot{u}(t)\}^{T}$

$G(t)$ is the control gain that is determined by minimizing the performance index $(J)$ using the PSO algorithm. The $J$ index is expressed as:

$J=\frac{1}{2} \int_{0}^{t}\left[u^{2} \cdot Q(1,1)+\dot{u}^{2} \cdot Q(2,1)+T_{\text {control }}(t) \cdot R\right] d t$

where $Q$ and $R$, the weight matrices that determine the importance of the structural responses and the control force respectively, are defined as:

$Q=\left[\begin{array}{cc}10^{8} & 0 \\ 0 & 10^{8}\end{array}\right], R=0.1$

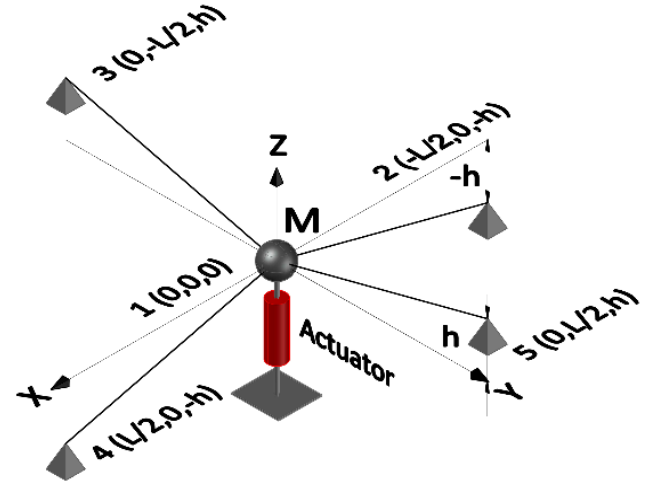

Figure 13 Geometric shape of equivalent SDOF cable net under the control force. 
A comparison between the displacement response of the controlled and uncontrolled cable nets under the wind excitation and the required control force are shown in Figure 14. The time history of the wind excitation is shown in Figure 3. The proposed control algorithm is able to reduce the maximum response up to $38 \%$ by applying a maximum control force of $3.7 \mathrm{kN}$, which proves the efficiency of the applied control algorithm. The parameters of the uncontrolled full FE model and the controlled equivalent SDOF model are presented in Table 3.
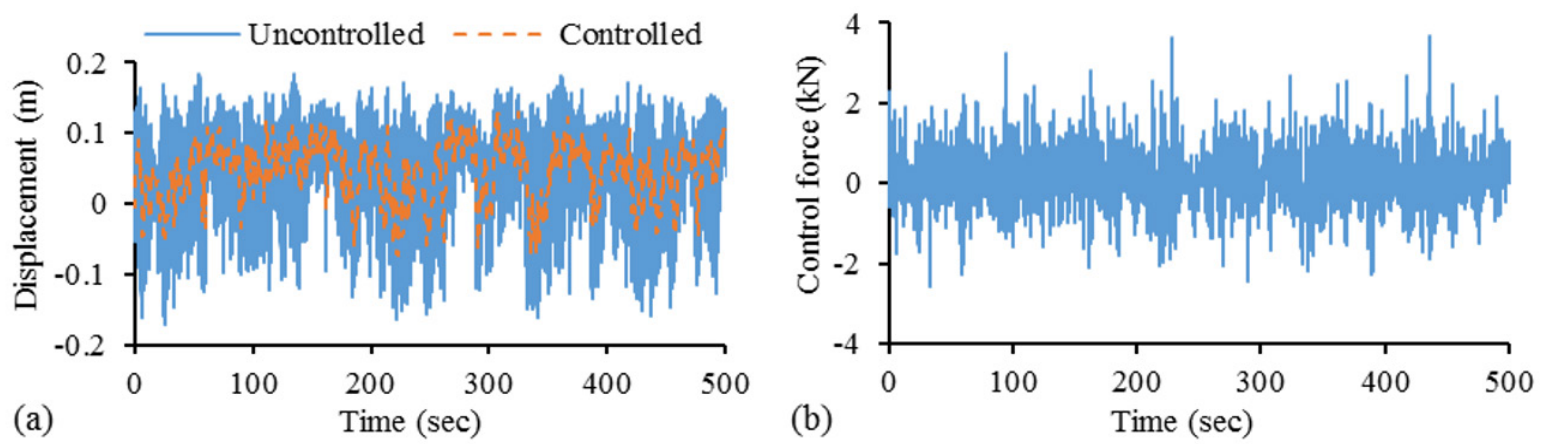

Figure 14 (a) Comparison of the displacement response of the controlled and uncontrolled models under the wind excitation;

(b) required control force.

In order to investigate the efficiency of proposed control algorithm on different rises, a numerical example is assumed in which the cables of SDOF models have $E A=7.80 \times 10^{3} \mathrm{kN}, L=5 \sqrt{2} \mathrm{~m}, T_{0}=1 \mathrm{kN}, \varepsilon_{0}=1.28 \times 10^{-4}$, $M=100 \mathrm{~kg}, \zeta=0$ and $h=0,0.25,0.5,0.75,1,1.25,1.5,1.75,2 \mathrm{~m}$. The models are subjected to wind excitation with the time history presented in Figure 3. The amount of reduction in structural response of controlled models for different rises and their corresponding control forces are shown in Figure 15. The more the rise increases, the more the efficiency of the control algorithm decreases. This is due to the higher stiffness of the structure in vertical direction. Therefore, it is recommended to apply the proposed control system in less curved cable nets.

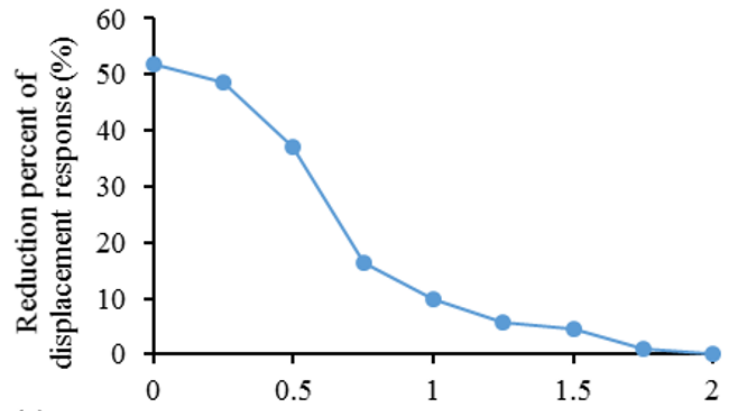

(a) $\mathrm{h}(\mathrm{m})$

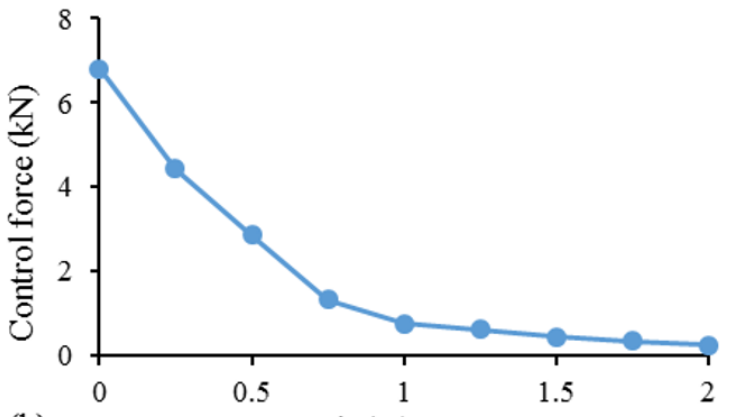

(b)

$\mathrm{h}(\mathrm{m})$

Figure 15 Efficiency of control algorithm for undamped cable nets with different rises under the wind excitation; (a) reduction percent of displacement response; (b) required control force.

\section{Conclusions}

The dynamic behavior of a saddle-shaped cable net with two high points and two low points was studied. The effects of the different parameters such as: mass and geometry of the structure, pretension and alignment of the cables and amplitude of the oscillation was investigated. An equivalent SDOF system was introduced to estimate the dynamic response of the full FE cable net and a nonlinear active control algorithm was proposed to reduce the structural responses. The following results have been obtained:

- $\quad$ The more the ratio of pretension in the edge cable to the net cable increases, the more the frequency of the cable net decreases. Because, the governed geometry obtain from form-finding analysis leads to both less sag in the edge cables and the bigger length of the net cables, consequently the stiffness of entire structure reduces.

- The effectiveness of increasing rise $(h)$ on the improvement of dynamic behavior strongly depends on the alignment of the net cables. The more the number of cables with higher rise increases, the less the response of the structure will be under the same load. This can be actualized by spanning the middle net cables between similar supports. 
- By creating curvature in the cable net, the higher modes are activated and compensate the effect of the first modes. Consequently, the vibrational amplitude of the structure drops considerably, which is called "the cancellation mode".

- The cable switching response spectrum is plotted. It is observed that the effect of the geometrically nonlinear behavior of the cable-net structures plays an important role, especially when the amplitude of the oscillation is increased. It results in higher eigen-frequency of the same cable-net under the similar conditions.

- Dynamic response factor in curved cable nets due to their high stiffness is less than 1 , but in flat structures is higher than 1 , which indicates the importance of dynamic analysis of flat cable-net structures.

- The results showed that the equivalent SDOF system as a simplified method can estimate the dynamic response of the MDOF flat model with $2.76 \%$ deviation. Therefore, the proposed solution can reduce the high cost of geometric nonlinear dynamics analysis.

- One way to reduce the displacement of the structure is to increase the pretension on the cables. However, this solution results in higher design forces of the cables as the side effect which rises the total costs. Therefore, a proposed nonlinear active control algorithm is applied as a solution to reduce the structural responses without raising the cable force. The efficiency of the proposed control algorithm has been proven in different cases by reducing the displacement up to $38 \%$. The results showed that the control algorithm works more efficiently in lower curved cable-nets.

Author's Contributions: Conceptualization, R Zarfam; Methodology, M Khalkhaliha and R Zarfam; Investigation, M Khalkhaliha; Writing - original draft, M Khalkhaliha; Writing - review \& editing, M Khalkhaliha; Funding acquisition, M Khalkhaliha; Re-sources, M Khalkhaliha and R Zarfam; Supervision, P Zarfam.

Editor: Jan Awrejcewicz.

\section{References}

Atai, A.A. and Mioduchowski, A. (1998). Equilibrium analysis of elasto-plastic cable nets. Computers \& Structures 66(2-3): 163171. doi: 10.1016/S0045-7949(97)00082-5.

Birnstiel, C. (1972). Analysis and design of cable structures. Computers \& Structures 2(5-6): 817-831. doi: 10.1016/00457949(72)90040-5.

Bleicher, A., Schlaich, M., Funio, Y. and Schauer, T. (2011). Model-based design and experimental of active vibration control for a stress ribbon bridge using pneumatic muscle actuators. Engineering Structures 33(8): 2237-2247. doi:

10.1016/j.engstruct.2011.02.035.

Bletzinger, K.U. and Ramm, E. (2001). Structural optimization and form finding of light weight structures. Computers \& Structures 79(22-25): 2053-2062. doi: 10.1016/S0045-7949(01)00052-9.

EN 1991-1-1 (2002), Eurocode 1: Actions on structures - Part 1-1: General actions - Densities, self-weight, imposed loads for buildings, European Standard.

ETA-11/0160, European Technical Assessment, PFEIFER Tension Members (2015). on the world wide web: https://www.pfeifer.info/out/assets/PFEIFER_TENSION-MEMBERS_BROCHURE_EN.PDF

Feng, R., Ye, J., Yan, G. and Ge, J. (2013). Dynamic Nonlinearity and Nonlinear Single-Degree-of-Freedom Model for Cable Net Glazing. Journal of Engineering Mechanics 139(10): 1446-1459. doi: 10.1061/(ASCE)EM.1943-7889.0000575.

Gambhir, M.L. and Batchelor, B. (1977). A finite element for 3-D prestressed cablenets. International Journal for Numerical Methods in Engineering 11(11): 1699-718. doi:10.1002/nme.1620111106.

Gambhir, M.L. and Batchelor, B. (1979). Finite element study of the free vibration of 3-D cable networks. International Journal of Solids and Structures 15(2): 127-136. doi: 10.1016/0020-7683(79)90017-9. 
Haiyan, H., Qiang, T., Wei, Z., Dongping, J., Gengkai, H. and Yanping, S. (2013). Nonlinear dynamics and control of large deployable space structures composed of trusses and meshes. Advances in Mechanics 43(4): 390-414. doi: 10.6052/10000992-13-045.

Haug, E. and Powell, G.H. (1972). Analytical shape finding for cable nets. Proc. 1971 IASS Pacific Symposium Part II on TENSION STRUCTURES and SPACE FRAMES, Tokyo and Kyoto, pp. 83-92, Paper No. 1-5, Architectural Institute of Japan.

Jager, E.M. and Furu, J. (1996). The theory of singular perturbations, Elsevier Science B.V., The Netherlands.

Kevorkian, J. and Cole, J.D. (1996). Multiple scale and singular perturbation methods, Springer-Verlag, U.S.A.

Kreysizig, E. (2011). Advanced engineering mathematics, 10th Edition, John Wiley \& Sons, Inc., U.S.A.

Kung, G.C. and Pao, Y.H. (1972). Nonlinear flexural vibrations of a clamped circular plate. Journal of Applied Mechanics 39(4): 1050-1054. doi: 10.1115/1.3422827.

Kwan, A.S.K. (2000). Simple technique for calculating natural frequencies of geometrically nonlinear prestressed cable structures. Computers \& Structures 74(1): 41-50. doi: 10.1016/S0045-7949(98)00318-6.

Lazzari, M., Saetta, A.V. and Vitaliani, R.V. (2001). Non-linear dynamic analysis of cable-suspended structures subjected to wind actions. Computers \& Structures 79(9): 953-969. doi: 10.1016/S0045-7949(00)00187-5.

Levy, R. and Spillers, W.R. (2003). Analysis of Geometrically Nonlinear Structures. 2nd ed., Kluwer Academic Publishers, The Netherlands.

McKenna, F., Fenves, G.L., Scott, M.H. and et al. (2000). Open System for Earthquake Engineering Simulation, OpenSees, University of California, Berkeley. CA.

Nayfeh, A. and Mook, D.T. (1979). Nonlinear oscillations, John Wiley \& Sons, Inc., U.S.A.

Papadrakakis, M. and Gantes, C.J. (1988). Truncated Newton methods for nonlinear finite element analysis. Computers \& Structures 30(3): 705-714. doi: 10.1016/0045-7949(88)90306-9.

Papadrakakis, M. and Balopoulos, V. (1991). Improved quasi-Newton methods for large nonlinear problems. Journal of Engineering Mechanics 117(6): 1201-1219. doi: 10.1061/(ASCE)0733-9399(1991)117:6(1201).

Parzygnat, W.J. and Pao, Y.H. (1978). Resonance phenomena in the non-linear vibration of plates governed by Dulling's equation. International Journal of Engineering Science 19: 966-1017.

Rand, R.H. (2005). Lecture notes on nonlinear vibrations, v.52, Cornell University, U.S.A.

Setio, S., Setio, H.D. and Jezequel, L. (1992a). A method of non-linear modal identification from frequency response tests. Journal of Sound and Vibration 158(3): 497-515. doi: 10.1016/0022-460X(92)90421-S.

Setio, S., Setio, H.D. and Jezequel, L. (1992b). Modal Analysis of Nonlinear Multi-degree-of-freedom Structures. International Jornal of Analytical and Experimental Modal Analysis 7(2): 75-93.

Shi, Y., Wu, L., Wang, Y., Lou, K. and Xu, Y. (2007). FEM Analysis and Experimental Study on Monolayer Cable Net for Glass Facades: Dynamic Properties. Advances in Structural Engineering 10(4): 383-395. doi: 10.1260/136943307783239327.

Soong, T.T. (1990). Active Structural Control: Theory and Practice, Longman, England and Wiley, New York.

Stefanou, G.D. (1992). Dynamic response of tension cable structures due to wind loads. Computers \& Structures 43(2): $365-$ 372. doi: 10.1016/0045-7949(92)90154-R.

Talvik, I. (2001). Finite element modelling of cable networks with flexible supports. Computers \& Structures 79(26): 24432450. doi: 10.1016/S0045-7949(01)00077-3.

Vakakis, A. (2002). Introduction to dynamical systems, Lecture Notes, National Technical University of Athens, Greece.

Vassilopoulou, I. and Gantes, C.J. (2005). Cable Nets with Elastically Deformable Edge Ring. International Journal of Space Structures 20(1): 15-34. doi: 10.1260/0266351054214353.

Vassilopoulou, I. and Gantes, C.J. (2010). Vibration modes and natural frequencies of saddle form cable nets. Computers \& Structures 88(1-2): 105-119. doi: 10.1016/j.compstruc.2009.07.002. 
Vassilopoulou, I. and Gantes, C.J. (2011). Nonlinear dynamic behavior of saddle-form cable nets under uniform harmonic load. Engineering Structures 33(10): 2762-2771. doi: 10.1016/j.engstruct.2011.06.001.

Vassilopoulou, I. and Gantes, C.J. (2012). Nonlinear dynamic phenomena in a SDOF model of cable net. Archive of Applied Mechanics 82(10-11): 1689- 1703. doi: 10.1007/s00419-012-0660-2.

Vassilopoulou, I. and Gantes, C.J. (2013). Nonlinear Dynamic Response of MDOF Cable Nets Estimated by Equivalent SDOF Models. Computational Methods in Earthquake Engineering 2: 345-379. doi: 10.1007/978-94-007-6573-3_17.

Vassilopoulou, I. and Gantes, C.J. (2016). Influence of a Deformable Contour Ring on the Nonlinear Dynamic Response of Cable Nets. Journal of Structures 6: 146-158. doi: 10.1016/j.istruc.2016.02.007.

Waziri, M.Y., Leong, W.J., Hassan, M.A. and Monsi, M. (2010). Jacobian computation-free Newton's method for systems of nonlinear equations. Journal of Numerical Mathematics and Stochastics 2(1): 54-63.

Wu, L., Shi, Y. and Wang, Y. (2010). Wind-Induced Response Characteristics of Monolayer Cable Net. Journal of Engineering Mechanics 136(3): 311-321. doi: 10.1061/(ASCE)0733-9399(2010)136:3(311).

Wu, Y.S., Zhang, W.Z., Meng, X.Y. and Su, Y. (2016). Nonlinear vibration control of cable net structures with bounded uncertainties. Acta Mechanica 227(10): 2985-3000. doi: 10.1007/s00707-016-1656-8.

Zarfam, R., Bletzinger, K.U. and Off, R. (2017). On the Dynamic of the Pre-stressed Flat Membrane Structures. Institute for Membrane and Shell technology, Anhalt University of Applied Science, Dessau, Germany.

Zingoni, A. (1996). An efficient computational scheme for the vibration analysis of high tension cable nets. Journal of Sound and Vibration 189(1): 55-79. doi: 10.1006/jsvi.1996.0005. 